\title{
Carbene Stabilization by Aryl Substituents. Is Bigger Better?
}

\author{
H. Lee Woodcock, * Damian Moran, *广乡 Bernard R. Brooks, ${ }^{*}$ \\ Paul v. R. Schleyer*t and Henry F. Schaefer III ${ }^{\dagger}$ \\ ${ }^{\dagger}$ Center for Computational Chemistry, University of Georgia, Athens, Georgia 30602-2525 \\ U.S.A, ${ }^{\ddagger}$ Computational Biophysics Section, Laboratory of Computational Biology, \\ National Heart Lung and Blood Institute, National Institutes of Health, Bethesda, MD \\ 20892 U.S.A., ${ }^{5}$ School of Chemistry, University of Sydney, Sydney, NSW 2006 Australia

\section{Supporting Information}

\section{Pages S1-S9, Complete References 57 and 60, Tables S1-S6}

*Corresponding authors: hlwood@nih.gov;dmoran@chem.usyd.edu.au;

schleyer@chem.uga.edu 


\section{Complete References 57 and 60}

(57) Shao, Y.; Fusti-Molnar, L.; Jung, Y.; Kussmann, J.; Ochsenfeld, C.; Brown, S. T.; Gilbert, A. T. B.; Slipchenko, L. V.; Levchenko, S. V.; O'Neill, D. P.; Distasio, R. A.; Lochan, R. C.; Wang, T.; Beran, G. J. O.; Besley, N. A.; Herbert, J. M.; Lin, C. Y.; Voorhis, T. V.; Chien, S. H.; Sodt, A.; Steele, R. P.; Rassolov, V. A.; Maslen, P. E.; Korambath, P. P.; Adamson, R. D.; Austin, B.; Baker, J.; Byrd, E. F. C.; Dachsel, H.; Doerksen, R. J.; Dreuw, A.; Dunietz, B. D.; Dutoi, A. D.; Furlani, T. R.; Gwaltney, S. R.; Heyden, A.; Hirata, S.; Hsu, C.-P.; Kedziora, G.; Khalliulin, R. Z.; Klunzinger, P.; Lee, A. M.; Lee, M. S.; Liang, W.; Lotan, I.; Nair, N.; Peters, B.; Proynov, E. I.; Pieniazek, P. A.; Rhee, Y. M.; Ritchie, J.; Rosta, E.; Sherrill, C. D.; Simmonett, A. C.; Subotnik, J. E.; Woodcock, H. L.; Zhang, W.; Bell, A. T.; Chakraborty, A. K.; Chipman, D. M.; Keil, F. J.; Warshel, A.; Hehre, W. J.; Schaefer, H. F.; Kong, J.; Krylov, A. I.; Gill, P. M. W.; HeadGordon, M. Phys. Chem. Chem. Phys. 2006, 8, 3172-3191.

(60) Frisch, M. J.; Trucks, G. W.; Schlegel, H. B.; Scuseria, G. E.; Robb, M. A.; Cheeseman, J. R.; Montgomery, J. A.; Vreven, T.; Kudin, K. N.; Burant, J. C.; Millam, J. M.; Iyengar, S. S.; Tomasi, J.; Barone, V.; Mennucci, B.; Cossi, M.; Scalmani, G.; Rega, N.; Petersson, G. A.; Nakatsuji, H.; Hada, M.; Ehara, M.; Toyota, K.; Fukuda, R.; Hasegawa, J.; Ishida, M.; Nakajima, T.; Honda, Y.; Kitao, O.; Nakai, H.; Klene, M.; Li, X.; Knox, J. E.; Hratchian, H. P.; Cross, J. B.; Bakken, V.; Adamo, C.; Jaramillo, J.; Gomperts, R.; Stratmann, R. E.; Yazyev, O.; Austin, A. J.; Cammi, R.; Pomelli, C.; Ochterski, J. W.; Ayala, P. Y.; Morokuma, K.; Voth, G. A.; Salvador, P.; Dannenberg, J. J.; Zakrzewski, V. G.; Dapprich, S.; Daniels, A. D.; Strain, M. C.; Farkas, O.; Malick, D. K.; Rabuck, A. D.; Raghavachari, K.; Foresman, J. B.; Ortiz, J. V.; Cui, Q.; Baboul, A. G.; Clifford, S.; Cioslowski, J.; Stefanov, B. B.; Liu, G.; Liashenko, A.; Piskorz, P.; Komaromi, I.; Martin, R. L.; Fox, D. J.; Keith, T.; Al-Laham, M. A.; Peng, C. Y.; Nanayakkara, A.; Challacombe, M.; Gill, P. M. W.; Johnson, B.; Chen, W.; Wong, M. W.; Gonzalez, C.; Pople, J. A.; Gaussian 03, revision C.02; Gaussian, Inc.: Wallingford CT, 2004.

Table S1. B3LYP/6-311+G(d,p) absolute energies and zero-point vibrational energies.

\begin{tabular}{|c|c|c|c|c|}
\hline & $\begin{array}{c}\text { Singlet } \\
(\mathrm{au})\end{array}$ & $\begin{array}{c}\text { ZPVE } \\
(\mathrm{kcal} / \mathrm{mol})\end{array}$ & $\begin{array}{c}\text { Triplet } \\
(\mathrm{au})\end{array}$ & $\begin{array}{c}\text { ZPVE } \\
(\mathrm{kcal} / \mathrm{mol})\end{array}$ \\
\hline Methylene & -39.14669 & 10.52 & -39.16608 & 10.81 \\
\hline Phenylcarbene & -270.29208 & 64.10 & -270.30032 & 63.67 \\
\hline Diphenylcarbene & -501.42157 & 116.22 & -501.42949 & 115.41 \\
\hline Napthylcarbene & -423.97181 & 93.73 & -423.98069 & 93.06 \\
\hline Dinapthylcarbene & -808.78150 & 175.21 & -808.78969 & 173.77 \\
\hline Anthrylcarbene & -577.64389 & 122.66 & -577.65635 & 121.97 \\
Dianthrylcarbene & -1116.12424 & 233.26 & -1116.14814 & 232.55 \\
\hline
\end{tabular}


Table S2. B3LYP/6-311+G(d,p) absolute energies and zero-point vibrational energies of the species used to compute isodesmic relative energies.

\begin{tabular}{|l|c|c|c|c|c|}
\hline & Energy (au) & ZPVE (kcal/mol) & & Energy (au) & ZPVE (kcal/mol) \\
\hline Methane & -40.53397 & 27.99 & 1-Methylnapthylene & -425.31550 & 110.42 \\
\hline Toluene & -271.63883 & 80.50 & 9-Methylanthracene & -578.98181 & 139.65 \\
\hline
\end{tabular}

Table S3. B3LYP/6-311+G(d,p) absolute energies and zero-point vibrational energies of the rotated transition structures 3TS, 5TS and 7TS.

\begin{tabular}{|c|c|c|c|c|c|}
\hline & Energy (au) & $\begin{array}{c}\text { ZPVE } \\
(\mathrm{kcal} / \mathrm{mol})\end{array}$ & & Energy (au) & $\begin{array}{c}\text { ZPVE } \\
(\mathrm{kcal} / \mathrm{mol})\end{array}$ \\
\hline Phenylcarbene & & & 9 -Anthrylcarbene & & \\
\hline${ }^{1}$ 3TS & -270.27112 & 62.55 & ${ }^{1} \mathbf{7 T S}$ & -577.61912 & 120.75 \\
\hline${ }^{3}$ 3TS & -270.29363 & 62.76 & ${ }^{3} \mathbf{7 T S}$ & -577.65008 & 120.57 \\
\hline 1-Napthylcarbene & & & & & \\
\hline${ }^{1}$ 5TS & -423.94839 & 91.92 & & & \\
\hline${ }^{3}$ 5TS & -423.97313 & 91.89 & & & \\
\hline
\end{tabular}

Table S4. B3LYP/6-311+G(d,p) absolute energies and zero-point vibrational energies of aryl carbenes with the singlet arylcarbene geometries constrained to the bond angles and lengths in singlet methylene and triplet arylcarbene geometries constrained to the bond angles and lengths in triplet methylene.

\begin{tabular}{|c|c|c|c|}
\hline Phenylcarbene & Energy (au) & Di(1-napthyl)carbene & Energy (au) \\
\hline Singlet & -270.29116 & Singlet & -808.76553 \\
\hline Triplet & -270.30032 & Triplet & -808.78759 \\
\hline Diphenylcarbene & & 9-Anthrylcarbene & -577.64192 \\
\hline Singlet & -501.40776 & Singlet & -577.65618 \\
\hline Triplet & -501.42837 & Triplet & -1116.09471 \\
\hline 1-Napthylcarbene & & Di(9-anthryl)carbene & -1116.13409 \\
\hline Singlet & -423.97095 & Singlet & Triplet \\
\hline Triplet & -423.98069 & & \\
\hline
\end{tabular}


Table S5. Singlet and triplet B3LYP/6-31G(d) optimized Cartesian coordinates (Å).

\begin{tabular}{|c|c|c|c|c|c|c|c|}
\hline \multicolumn{4}{|c|}{ Di(9-anthryl)carbene singlet } & \multicolumn{4}{|c|}{ yl)carbene triplet } \\
\hline $\mathrm{H}$ & -2.718068 & 3.679646 & 3.486627 & $\mathrm{H}$ & 4.121972 & -3.251664 & 3.253654 \\
\hline $\mathrm{C}$ & -2.436139 & 2.962306 & 2.723984 & $\mathrm{C}$ & 3.564931 & -2.593930 & 2.595737 \\
\hline $\mathrm{H}$ & -4.451911 & 2.436555 & 2.243828 & $\mathrm{H}$ & 5.314517 & -1.721436 & 1.724460 \\
\hline $\mathrm{C}$ & -3.400083 & 2.269024 & 2.036689 & $\mathrm{C}$ & 4.229861 & -1.741640 & 1.744020 \\
\hline $\mathrm{C}$ & -.700924 & 1.846360 & 1.452645 & $\mathrm{C}$ & 1.440646 & -1.774785 & 1.775539 \\
\hline $\mathrm{C}$ & -3.049275 & 1.319893 & 1.032024 & $\mathrm{C}$ & 3.519287 & -.870811 & .872967 \\
\hline $\mathrm{C}$ & -1.071223 & 2.741165 & 2.435501 & $\mathrm{C}$ & 2.156582 & -2.611562 & 2.612557 \\
\hline $\mathrm{C}$ & -1.662204 & 1.112227 & .726829 & $\mathrm{C}$ & 2.091867 & -.893060 & .894368 \\
\hline $\mathrm{C}$ & -4.036156 & .570669 & .372215 & $\mathrm{C}$ & 4.202977 & .003209 & -.000397 \\
\hline $\mathrm{H}$ & -.309382 & 3.280555 & 2.986613 & $\mathrm{H}$ & 1.631879 & -3.282533 & 3.283103 \\
\hline $\mathrm{C}$ & .000000 & .000000 & -.721475 & $\mathrm{C}$ & .000000 & .000000 & .000126 \\
\hline $\mathrm{H}$ & .348537 & 1.707181 & 1.227513 & $\mathrm{H}$ & .356815 & -1.793695 & 1.793839 \\
\hline $\mathrm{C}$ & -3.722273 & -.348683 & -.641835 & $\mathrm{C}$ & 3.517742 & .876274 & -.873513 \\
\hline $\mathrm{H}$ & -5.075111 & .707612 & .654922 & $\mathrm{H}$ & 5.287924 & .003994 & -.000569 \\
\hline $\mathrm{C}$ & -4.737013 & -1.111604 & -1.290567 & $\mathrm{C}$ & 4.226786 & 1.748256 & -1.744652 \\
\hline $\mathrm{C}$ & -2.361441 & -.514779 & -1.054873 & $\mathrm{C}$ & 2.090282 & .896419 & -.894525 \\
\hline $\mathrm{H}$ & -1.056734 & -1.482125 & -2.459350 & $\mathrm{H}$ & .353631 & 1.794654 & -1.793326 \\
\hline $\mathrm{C}$ & -1.291330 & .178647 & -.338836 & $\mathrm{C}$ & 1.336075 & .001055 & -.000068 \\
\hline C & -4.428861 & -1.968704 & -2.317223 & $\mathrm{C}$ & 3.560353 & 2.599680 & -2.596064 \\
\hline $\mathrm{H}$ & -5.762770 & -1.000497 & -.954850 & $\mathrm{H}$ & 5.311474 & 1.729654 & -1.725395 \\
\hline $\mathrm{H}$ & -5.210853 & -2.540200 & -2.804652 & $\mathrm{H}$ & 4.116232 & 3.258327 & -3.254050 \\
\hline $\mathrm{C}$ & -3.090496 & -2.091918 & -2.755120 & $\mathrm{C}$ & 2.151974 & 2.615256 & -2.612470 \\
\hline $\mathrm{H}$ & -2.856222 & -2.736276 & -3.594971 & $\mathrm{H}$ & 1.626099 & 3.285575 & -3.282754 \\
\hline $\mathrm{C}$ & -2.084618 & -1.387537 & -2.127388 & $\mathrm{C}$ & 1.437498 & 1.777313 & -1.775370 \\
\hline $\mathrm{C}$ & 1.291330 & -.178647 & -.338836 & $\mathrm{C}$ & -1.336075 & -.001055 & -.000068 \\
\hline $\mathrm{H}$ & 2.856222 & 2.736276 & -3.594971 & $\mathrm{H}$ & -1.626099 & -3.285575 & -3.282754 \\
\hline $\mathrm{C}$ & 3.090496 & 2.091918 & -2.755120 & $\mathrm{C}$ & -2.151974 & -2.615256 & -2.612470 \\
\hline $\mathrm{H}$ & 1.056734 & 1.482125 & -2.459350 & $\mathrm{H}$ & -.353631 & -1.794654 & -1.793326 \\
\hline $\mathrm{C}$ & 2.084618 & 1.387537 & -2.127388 & $\mathrm{C}$ & -1.437498 & -1.777313 & -1.775370 \\
\hline C & 4.737013 & 1.111604 & -1.290567 & $\mathrm{C}$ & -4.226786 & -1.748256 & -1.744652 \\
\hline $\mathrm{C}$ & 2.361441 & .514779 & -1.054873 & $\mathrm{C}$ & -2.090282 & -.896419 & -.894525 \\
\hline $\mathrm{C}$ & 4.428861 & 1.968704 & -2.317223 & $\mathrm{C}$ & -3.560353 & -2.599680 & -2.596064 \\
\hline C & 3.722273 & .348683 & -.641835 & $\mathrm{C}$ & -3.517742 & -.876274 & -.873513 \\
\hline $\mathrm{H}$ & 5.210853 & 2.540200 & -2.804652 & $\mathrm{H}$ & -4.116232 & -3.258327 & -3.254050 \\
\hline $\mathrm{H}$ & 5.075111 & -.707612 & .654922 & $\mathrm{H}$ & -5.287924 & -.003994 & -.000569 \\
\hline $\mathrm{H}$ & 5.762770 & 1.000497 & -.954850 & $\mathrm{H}$ & -5.311474 & -1.729654 & -1.725395 \\
\hline $\mathrm{C}$ & 1.662204 & -1.112227 & .726829 & $\mathrm{C}$ & -2.091867 & .893060 & .894368 \\
\hline C & .700924 & -1.846360 & 1.452645 & $\mathrm{C}$ & -1.440646 & 1.774785 & 1.775539 \\
\hline C & 3.049275 & -1.319893 & 1.032024 & $\mathrm{C}$ & -3.519287 & .870811 & .872967 \\
\hline $\mathrm{H}$ & 4.451911 & -2.436555 & 2.243828 & $\mathrm{H}$ & -5.314517 & 1.721436 & 1.724460 \\
\hline $\mathrm{C}$ & 4.036156 & -.570669 & .372215 & $\mathrm{C}$ & -4.202977 & -.003209 & -.000397 \\
\hline $\mathrm{C}$ & 1.071223 & -2.741165 & 2.435501 & $\mathrm{C}$ & -2.156582 & 2.611562 & 2.612557 \\
\hline $\mathrm{H}$ & -.348537 & -1.707181 & 1.227513 & $\mathrm{H}$ & -.356815 & 1.793695 & 1.793839 \\
\hline $\mathrm{H}$ & .309382 & -3.280555 & 2.986613 & $\mathrm{H}$ & -1.631879 & 3.282533 & 3.283103 \\
\hline $\mathrm{C}$ & 2.436139 & -2.962306 & 2.723984 & $\mathrm{C}$ & -3.564931 & 2.593930 & 2.595737 \\
\hline $\mathrm{H}$ & 2.718068 & -3.679646 & 3.486627 & $\mathrm{H}$ & -4.121972 & 3.251664 & 3.253654 \\
\hline $\mathrm{C}$ & 3.400083 & -2.269024 & 2.036689 & $\mathrm{C}$ & -4.229861 & 1.741640 & 1.744020 \\
\hline
\end{tabular}




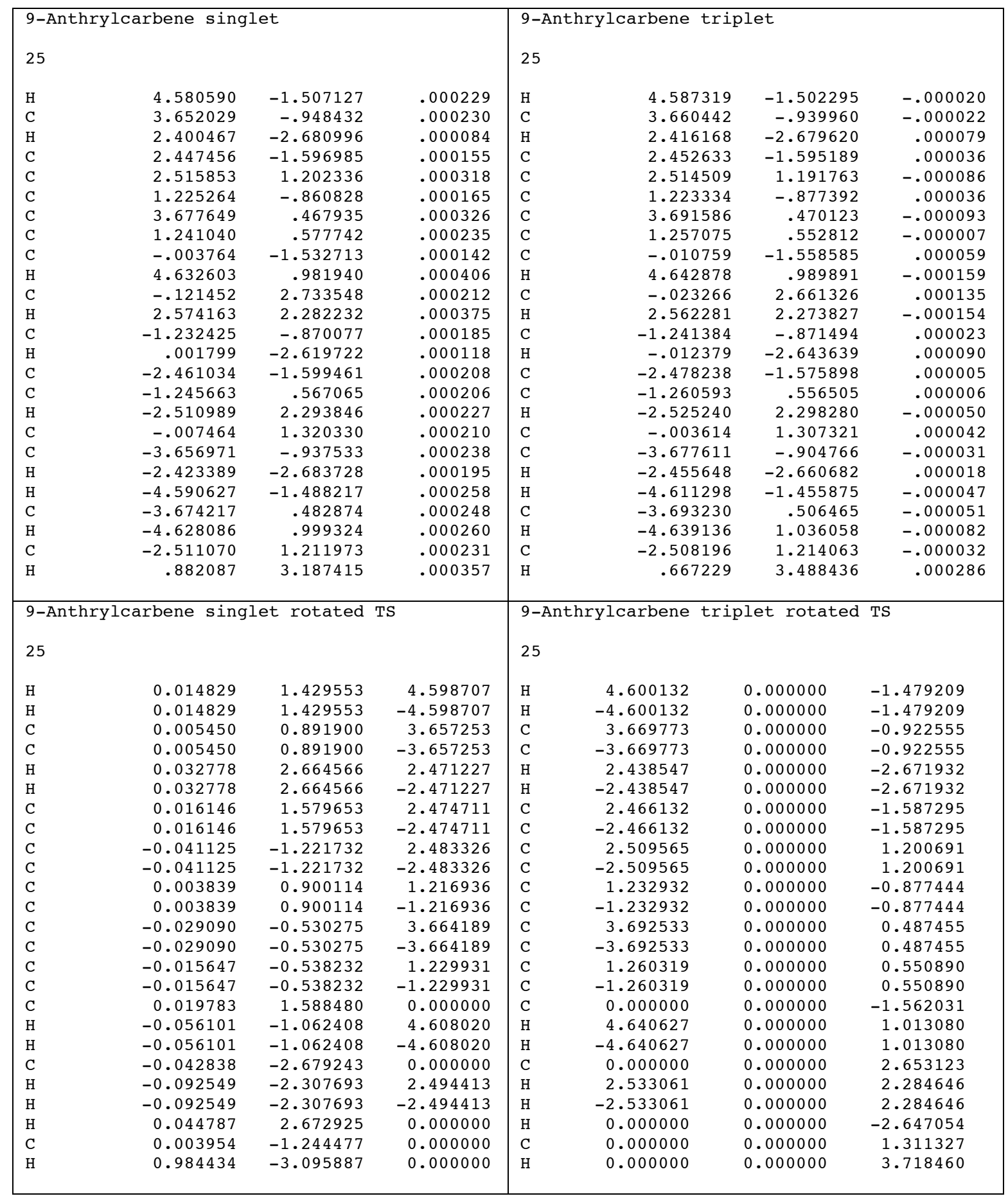




\begin{tabular}{|c|c|c|c|c|c|c|c|}
\hline \multicolumn{4}{|c|}{ Di(1-napthyl)carbene singlet } & $\mathrm{Di}$ & \multicolumn{3}{|c|}{ Di(1-napthyl)carbene triplet } \\
\hline $\mathrm{H}$ & 6.003244 & 1.063804 & -1.683292 & $\mathrm{H}$ & 5.108645 & 1.842316 & -2.326688 \\
\hline $\mathrm{H}$ & -6.003244 & -1.063804 & -1.683292 & $\mathrm{H}$ & -5.108645 & -1.842316 & -2.326688 \\
\hline $\mathrm{C}$ & 5.032997 & 0.771131 & -1.297748 & $\mathrm{C}$ & 4.341739 & 1.393562 & -1.705432 \\
\hline $\mathrm{C}$ & -5.032997 & -0.771131 & -1.297748 & $\mathrm{C}$ & -4.341739 & -1.393562 & -1.705432 \\
\hline $\mathrm{H}$ & 5.842377 & -0.366013 & 0.325934 & $\mathrm{H}$ & 5.700008 & 0.070241 & -0.711374 \\
\hline $\mathrm{H}$ & -5.842377 & 0.366013 & 0.325934 & $\mathrm{H}$ & -5.700008 & -0.070241 & -0.711374 \\
\hline $\mathrm{C}$ & 4.944579 & -0.024908 & -0.179217 & $\mathrm{C}$ & 4.672042 & 0.405006 & -0.804342 \\
\hline $\mathrm{C}$ & -4.944579 & 0.024908 & -0.179217 & $\mathrm{C}$ & -4.672042 & -0.405006 & -0.804342 \\
\hline $\mathrm{C}$ & 2.617511 & 0.841232 & -1.470060 & $\mathrm{C}$ & 2.024105 & 1.253042 & -1.030938 \\
\hline $\mathrm{C}$ & -2.617511 & -0.841232 & -1.470060 & $\mathrm{C}$ & -2.024105 & -1.253042 & -1.030938 \\
\hline $\mathrm{C}$ & 3.681172 & -0.416314 & 0.333964 & $\mathrm{C}$ & 3.685590 & -0.195446 & 0.017787 \\
\hline $\mathrm{C}$ & -3.681172 & 0.416314 & 0.333964 & $\mathrm{C}$ & -3.685590 & 0.195446 & 0.017787 \\
\hline $\mathrm{C}$ & 3.858611 & 1.196508 & -1.954328 & $\mathrm{C}$ & 3.005498 & 1.821643 & -1.820033 \\
\hline $\mathrm{C}$ & -3.858611 & -1.196508 & -1.954328 & $\mathrm{C}$ & -3.005498 & -1.821643 & -1.820033 \\
\hline $\mathrm{C}$ & 2.494020 & 0.037992 & -0.315611 & $\mathrm{C}$ & 2.334125 & 0.242105 & -0.100923 \\
\hline $\mathrm{C}$ & -2.494020 & -0.037992 & -0.315611 & $\mathrm{C}$ & -2.334125 & -0.242105 & -0.100923 \\
\hline $\mathrm{C}$ & 3.572163 & -1.264864 & 1.469556 & $\mathrm{C}$ & 4.020957 & -1.218540 & 0.951388 \\
\hline $\mathrm{C}$ & -3.572163 & 1.264864 & 1.469556 & $\mathrm{C}$ & -4.020957 & 1.218540 & 0.951388 \\
\hline $\mathrm{H}$ & 3.935300 & 1.800826 & -2.851520 & $\mathrm{H}$ & 2.745820 & 2.598863 & -2.529769 \\
\hline $\mathrm{H}$ & -3.935300 & -1.800826 & -2.851520 & $\mathrm{H}$ & -2.745820 & -2.598863 & -2.529769 \\
\hline $\mathrm{C}$ & 0.000000 & 0.000000 & -0.450266 & $\mathrm{C}$ & 0.000000 & 0.000000 & 0.673018 \\
\hline $\mathrm{H}$ & 1.710001 & 1.155611 & -1.971690 & $\mathrm{H}$ & 0.995497 & 1.584045 & -1.121843 \\
\hline $\mathrm{H}$ & -1.710001 & -1.155611 & -1.971690 & $\mathrm{H}$ & -0.995497 & -1.584045 & -1.121843 \\
\hline $\mathrm{C}$ & 2.340695 & -1.626126 & 1.970949 & $\mathrm{C}$ & 3.044834 & -1.794561 & 1.748031 \\
\hline $\mathrm{C}$ & -2.340695 & 1.626126 & 1.970949 & $\mathrm{C}$ & -3.044834 & 1.794561 & 1.748031 \\
\hline $\mathrm{H}$ & 4.481451 & -1.629312 & 1.936639 & $\mathrm{H}$ & 5.053734 & -1.539616 & 1.028400 \\
\hline $\mathrm{H}$ & -4.481451 & 1.629312 & 1.936639 & $\mathrm{H}$ & -5.053734 & 1.539616 & 1.028400 \\
\hline $\mathrm{C}$ & 1.165160 & -1.120983 & 1.392930 & $\mathrm{C}$ & 1.714166 & -1.392685 & 1.660265 \\
\hline $\mathrm{C}$ & -1.165160 & 1.120983 & 1.392930 & $\mathrm{C}$ & -1.714166 & 1.392685 & 1.660265 \\
\hline $\mathrm{C}$ & 1.196105 & -0.311243 & 0.240185 & $\mathrm{C}$ & 1.303566 & -0.371227 & 0.748525 \\
\hline $\mathrm{C}$ & -1.196105 & 0.311243 & 0.240185 & $\mathrm{C}$ & -1.303566 & 0.371227 & 0.748525 \\
\hline $\mathrm{H}$ & -2.275400 & 2.281257 & 2.832770 & $\mathrm{H}$ & -3.315639 & 2.571955 & 2.454214 \\
\hline $\mathrm{H}$ & 2.275400 & -2.281257 & 2.832770 & $\mathrm{H}$ & 3.315639 & -2.571955 & 2.454214 \\
\hline $\mathrm{H}$ & -0.203122 & 1.399861 & 1.804769 & $\mathrm{H}$ & -0.962110 & 1.852760 & 2.289456 \\
\hline $\mathrm{H}$ & 0.203122 & -1.399861 & 1.804769 & $\mathrm{H}$ & 0.962110 & -1.852760 & 2.289456 \\
\hline \multicolumn{4}{|c|}{ 1-Napthylcarbene singlet } & \multicolumn{4}{|c|}{ 1-Napthylcarbene triplet } \\
\hline 19 & & & & 19 & & & \\
\hline $\mathrm{C}$ & 2.701133 & 0.355358 & 0.000000 & $\mathrm{C}$ & 2.705020 & 0.343500 & 0.000000 \\
\hline $\mathrm{C}$ & 1.699191 & 1.294689 & 0.000000 & $\mathrm{C}$ & 1.699193 & 1.283928 & 0.000000 \\
\hline $\mathrm{C}$ & 0.337536 & 0.891130 & 0.000000 & $\mathrm{C}$ & 0.335638 & 0.895502 & 0.000000 \\
\hline $\mathrm{C}$ & 0.011829 & -0.502026 & 0.000000 & $\mathrm{C}$ & 0.016416 & -0.494130 & 0.000000 \\
\hline $\mathrm{C}$ & 1.067062 & -1.441602 & 0.000000 & $\mathrm{C}$ & 1.067001 & -1.434769 & 0.000000 \\
\hline $\mathrm{C}$ & 2.378488 & -1.020871 & 0.000000 & $\mathrm{C}$ & 2.386030 & -1.029083 & 0.000000 \\
\hline $\mathrm{H}$ & -0.453486 & 2.896535 & 0.000000 & $\mathrm{H}$ & -0.454665 & 2.913585 & 0.000000 \\
\hline $\mathrm{H}$ & 3.739844 & 0.665697 & 0.000000 & $\mathrm{H}$ & 3.742986 & 0.657184 & 0.000000 \\
\hline $\mathrm{H}$ & 1.934096 & 2.353890 & 0.000000 & $\mathrm{H}$ & 1.939346 & 2.342177 & 0.000000 \\
\hline $\mathrm{C}$ & -0.712387 & 1.841916 & 0.000000 & $\mathrm{C}$ & -0.712077 & 1.860183 & 0.000000 \\
\hline $\mathrm{C}$ & -1.385000 & -0.909005 & 0.000000 & $\mathrm{C}$ & -1.384157 & -0.906951 & 0.000000 \\
\hline $\mathrm{H}$ & 0.802686 & -2.491242 & 0.000000 & $\mathrm{H}$ & 0.821574 & -2.491081 & 0.000000 \\
\hline $\mathrm{H}$ & 3.177603 & -1.754098 & 0.000000 & $\mathrm{H}$ & 3.179783 & -1.767671 & 0.000000 \\
\hline $\mathrm{C}$ & -2.365626 & 0.100780 & 0.000000 & $\mathrm{C}$ & -2.379888 & 0.109105 & 0.000000 \\
\hline $\mathrm{C}$ & -2.041025 & 1.462240 & 0.000000 & $\mathrm{C}$ & -2.036776 & 1.462240 & 0.000000 \\
\hline $\mathrm{H}$ & -2.823014 & 2.212833 & 0.000000 & $\mathrm{H}$ & -3.423375 & -0.184073 & 0.000000 \\
\hline $\mathrm{H}$ & -3.407432 & -0.202897 & 0.000000 & $\mathrm{H}$ & -2.824891 & 2.207472 & 0.000000 \\
\hline $\mathrm{C}$ & -1.718740 & -2.299291 & 0.000000 & $\mathrm{C}$ & -1.751026 & -2.233610 & 0.000000 \\
\hline $\mathrm{H}$ & -2.823267 & -2.361649 & 0.000000 & $\mathrm{H}$ & -2.686308 & -2.774113 & 0.000000 \\
\hline
\end{tabular}




\begin{tabular}{|c|c|c|c|c|c|c|c|}
\hline \multicolumn{4}{|c|}{ 1-Napthylcarbene singlet rotated TS } & $1-$ & arbene tri & et rotated & \\
\hline $\mathrm{C}$ & 0.004775 & -0.474309 & 0.004902 & $\mathrm{C}$ & 0.017963 & -0.467019 & 0.031863 \\
\hline $\mathrm{C}$ & -1.368951 & -0.899233 & 0.016824 & $\mathrm{C}$ & -1.376069 & -0.901070 & 0.066709 \\
\hline $\mathrm{C}$ & -1.704342 & -2.293728 & -0.069210 & $\mathrm{C}$ & -1.725351 & -2.243349 & 0.069682 \\
\hline $\mathrm{H}$ & -1.760975 & -2.730576 & 0.948343 & $\mathrm{H}$ & -1.944787 & -3.143976 & 0.611014 \\
\hline C & 2.677579 & 0.392485 & 0.007254 & $\mathrm{C}$ & 2.695179 & 0.415520 & -0.007455 \\
\hline $\mathrm{C}$ & 1.663589 & 1.320583 & 0.016388 & $\mathrm{C}$ & 1.675144 & 1.339120 & -0.015791 \\
\hline C & 0.300257 & 0.922884 & 0.016736 & $\mathrm{C}$ & 0.317590 & 0.928240 & 0.001485 \\
\hline C & 1.071342 & -1.410476 & -0.014403 & $\mathrm{C}$ & 1.086133 & -1.390040 & 0.032876 \\
\hline $\mathrm{C}$ & 2.379937 & -0.989288 & -0.009757 & $\mathrm{C}$ & 2.396958 & -0.962602 & 0.015185 \\
\hline $\mathrm{H}$ & -0.530020 & 2.922788 & 0.044707 & $\mathrm{H}$ & -0.502179 & 2.932120 & -0.038641 \\
\hline $\mathrm{H}$ & 3.711642 & 0.718651 & 0.007384 & $\mathrm{H}$ & 3.728181 & 0.744754 & -0.021371 \\
\hline $\mathrm{H}$ & 1.892913 & 2.381212 & 0.020927 & $\mathrm{H}$ & 1.897213 & 2.401100 & -0.037163 \\
\hline C & -0.761379 & 1.863908 & 0.028415 & $\mathrm{C}$ & -0.743810 & 1.875060 & -0.013162 \\
\hline $\mathrm{H}$ & 0.848413 & -2.473559 & -0.053243 & $\mathrm{H}$ & 0.858558 & -2.449998 & 0.040230 \\
\hline $\mathrm{H}$ & 3.185155 & -1.714805 & -0.025989 & $\mathrm{H}$ & 3.202768 & -1.688109 & 0.015140 \\
\hline $\mathrm{C}$ & -2.380956 & 0.056504 & -0.036588 & $\mathrm{C}$ & -2.378788 & 0.096410 & 0.033887 \\
\hline $\mathrm{C}$ & -2.064617 & 1.429819 & 0.003925 & $\mathrm{C}$ & -2.060024 & 1.458339 & 0.000151 \\
\hline $\mathrm{H}$ & -2.877851 & 2.147326 & 0.000097 & $\mathrm{H}$ & -2.860191 & 2.190249 & -0.013522 \\
\hline $\mathrm{H}$ & -3.418014 & -0.252523 & -0.094155 & $\mathrm{H}$ & -3.415991 & -0.217087 & 0.041441 \\
\hline \multicolumn{4}{|c|}{ Diphenylcarbene singlet } & \multicolumn{4}{|c|}{ Diphenylcarbene triplet } \\
\hline 23 & & & & 23 & & & \\
\hline $\mathrm{C}$ & 1.235685 & .039313 & -.565439 & $\mathrm{C}$ & 1.326209 & .013967 & -.398306 \\
\hline $\mathrm{C}$ & -1.235685 & -.039313 & -.565439 & $\mathrm{C}$ & -1.326209 & -.013967 & -.398306 \\
\hline $\mathrm{C}$ & .000000 & .000000 & -1.285933 & $\mathrm{C}$ & .000000 & .000000 & -.843649 \\
\hline $\mathrm{C}$ & 3.753564 & .134035 & .696681 & $\mathrm{C}$ & 4.012747 & .087843 & .478261 \\
\hline $\mathrm{C}$ & -3.753564 & -.134035 & .696681 & $\mathrm{C}$ & -4.012747 & -.087843 & .478261 \\
\hline C & 2.373717 & -.576027 & -1.148471 & $\mathrm{C}$ & 2.371504 & -.558764 & -1.176079 \\
\hline C & 1.415752 & .732414 & .662234 & $\mathrm{C}$ & -1.684641 & -.640348 & .832216 \\
\hline C & -2.373717 & .576027 & -1.148471 & $\mathrm{C}$ & -2.371504 & .558764 & -1.176079 \\
\hline $\mathrm{C}$ & -1.415752 & -.732414 & .662234 & $\mathrm{C}$ & 1.684641 & .640348 & .832216 \\
\hline C & 2.666095 & .803152 & 1.262277 & $\mathrm{C}$ & 3.003437 & .665761 & 1.255675 \\
\hline $\mathrm{C}$ & 3.599623 & -.570589 & -.502110 & $\mathrm{C}$ & -3.685110 & .520310 & -.738400 \\
\hline $\mathrm{C}$ & -2.666095 & -.803152 & 1.262277 & $\mathrm{C}$ & -3.003437 & -.665761 & 1.255675 \\
\hline $\mathrm{C}$ & -3.599623 & .570589 & -.502110 & $\mathrm{C}$ & 3.685110 & -.520310 & -.738400 \\
\hline $\mathrm{H}$ & 2.249179 & -1.063414 & -2.108293 & $\mathrm{H}$ & 2.119543 & -1.031148 & -2.118222 \\
\hline $\mathrm{H}$ & .571663 & 1.245601 & 1.107540 & $\mathrm{H}$ & -.908871 & -1.102913 & 1.430846 \\
\hline $\mathrm{H}$ & -2.249179 & 1.063414 & -2.108293 & $\mathrm{H}$ & -2.119543 & 1.031148 & -2.118222 \\
\hline $\mathrm{H}$ & -.571663 & -1.245601 & 1.107540 & $\mathrm{H}$ & .908871 & 1.102913 & 1.430846 \\
\hline $\mathrm{H}$ & 2.795829 & 1.365758 & 2.180552 & $\mathrm{H}$ & 3.253617 & 1.145040 & 2.196026 \\
\hline $\mathrm{H}$ & 4.450148 & -1.076521 & -.945287 & $\mathrm{H}$ & -4.464914 & .967073 & -1.345312 \\
\hline $\mathrm{H}$ & -2.795829 & -1.365758 & 2.180552 & $\mathrm{H}$ & -3.253617 & -1.145040 & 2.196026 \\
\hline $\mathrm{H}$ & -4.450148 & 1.076521 & -.945287 & $\mathrm{H}$ & 4.464914 & -.967073 & -1.345312 \\
\hline $\mathrm{H}$ & 4.723938 & .175486 & 1.179130 & $\mathrm{H}$ & 5.042685 & .114573 & .814723 \\
\hline $\mathrm{H}$ & -4.723938 & -.175486 & 1.179130 & $\mathrm{H}$ & -5.042685 & -.114573 & .814723 \\
\hline
\end{tabular}




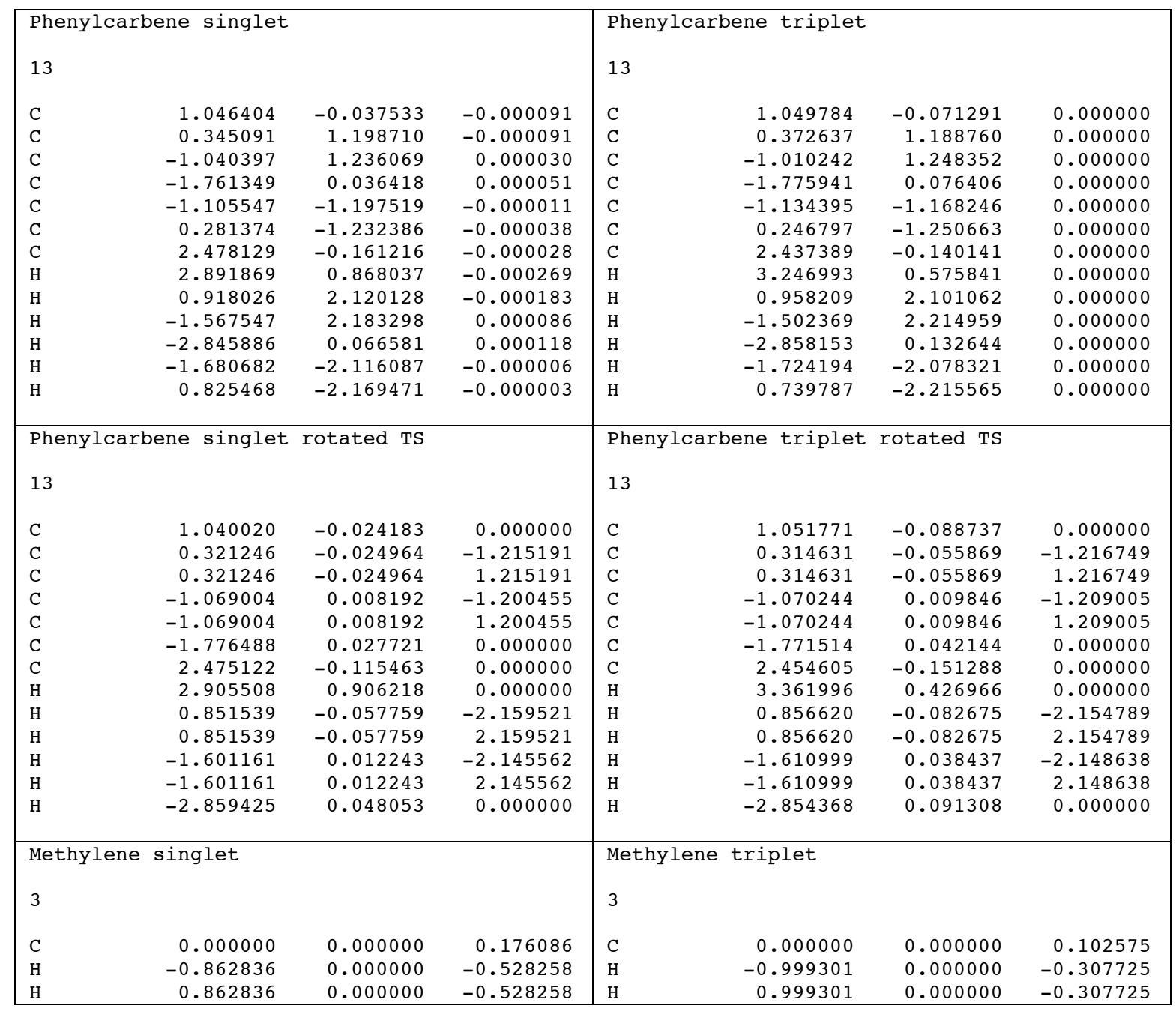


Table S6. B3LYP/6-31G(d) optimized Cartesian coordinates ( $)$ of di(9anthryl)diazomethane.

\begin{tabular}{|c|c|c|c|}
\hline 47 & & & \\
\hline H & 1.61018800 & 4.24525500 & -3.28325600 \\
\hline C & 1.55613300 & 3.45730400 & -2.53708900 \\
\hline $\mathrm{H}$ & 3.63967800 & 2.98355100 & -2.63866300 \\
\hline C & 2.67819100 & 2.75888700 & -2.18303500 \\
\hline C & 0.21788700 & 2.16329500 & -0.97376000 \\
\hline C & 2.62397500 & 1.72038300 & -1.20157100 \\
\hline C & 0.31304500 & 3.15723400 & -1.91368000 \\
\hline C & 1.35752400 & 1.38811200 & -0.58808700 \\
\hline C & 3.78199800 & 1.04554700 & -0.80680600 \\
\hline $\mathrm{H}$ & -0.57214100 & 3.72700300 & -2.18316800 \\
\hline C & -0.00000500 & 0.00002800 & 1.02460000 \\
\hline $\mathrm{H}$ & -0.73968700 & 1.96176400 & -0.51259700 \\
\hline C & 3.74793400 & 0.02772600 & 0.14909800 \\
\hline $\mathrm{H}$ & 4.73442100 & 1.31939100 & -1.25596800 \\
\hline C & 4.94138000 & -0.65701600 & 0.53850700 \\
\hline $\mathrm{C}$ & 2.48387800 & -0.35674600 & 0.73581400 \\
\hline $\mathrm{H}$ & 1.56070500 & -1.82426200 & 2.05972900 \\
\hline $\mathrm{C}$ & 1.29855500 & 0.34296800 & 0.37360700 \\
\hline C & 4.90577600 & -1.68996400 & 1.43525900 \\
\hline H & 5.88207100 & -0.33882300 & 0.09534300 \\
\hline $\mathrm{H}$ & 5.81951100 & -2.20297700 & 1.72203100 \\
\hline C & 3.65937300 & -2.10749900 & 1.97934600 \\
\hline $\mathrm{H}$ & 3.62962000 & -2.94911000 & 2.66593500 \\
\hline C & 2.49424800 & -1.46774800 & 1.64117700 \\
\hline C & -1.29856600 & -0.34293200 & 0.37362400 \\
\hline $\mathrm{H}$ & -3.62971100 & 2.94906900 & 2.66597900 \\
\hline $\mathrm{C}$ & -3.65944400 & 2.10746000 & 1.97938500 \\
\hline $\mathrm{H}$ & -1.56076700 & 1.82427800 & 2.05975900 \\
\hline $\mathrm{C}$ & -2.49430300 & 1.46774400 & 1.64120800 \\
\hline C & -4.94141700 & 0.65694400 & 0.53854900 \\
\hline $\mathrm{C}$ & -2.48390600 & 0.35674800 & 0.73583900 \\
\hline C & -4.90583800 & 1.68989200 & 1.43530300 \\
\hline C & -3.74795400 & -0.02776200 & 0.14913200 \\
\hline $\mathrm{H}$ & -5.81958600 & 2.20287800 & 1.72208100 \\
\hline $\mathrm{H}$ & -4.73441100 & -1.31946100 & -1.25592700 \\
\hline $\mathrm{H}$ & -5.88210200 & 0.33872400 & 0.09539100 \\
\hline C & -1.35751100 & -1.38808300 & -0.58805400 \\
\hline C & -0.21784900 & -2.16323100 & -0.97372000 \\
\hline C & -2.62395300 & -1.72039400 & -1.20153300 \\
\hline $\mathrm{H}$ & -3.63961700 & -2.98360500 & -2.63861400 \\
\hline C & -3.78199300 & -1.04558700 & -0.80677000 \\
\hline C & -0.31297700 & -3.15718100 & -1.91363100 \\
\hline $\mathrm{H}$ & 0.73971900 & -1.96166300 & -0.51255800 \\
\hline $\mathrm{H}$ & 0.57222600 & -3.72692600 & -2.18311600 \\
\hline C & -1.55605700 & -3.45729400 & -2.53703700 \\
\hline $\mathrm{H}$ & -1.61008900 & -4.24525300 & -3.28319700 \\
\hline C & -2.67813700 & -2.75890900 & -2.18298800 \\
\hline
\end{tabular}

